\title{
Shear wave elasticity imaging based on acoustic radiation force and optical detection
}

\author{
Yi Cheng ${ }^{1}$, Rui Li ${ }^{1}$, Sinan Li ${ }^{1}$, Christopher Dunsby ${ }^{2}$, Robert Eckersley ${ }^{3}$, Daniel \\ Elson $^{4}$, Meng-Xing Tang ${ }^{1}$
}

1 Department of Bioengineering, Imperial College London, Exhibition Road, London SW7 2AZ, UK

2 Department of Physics, Imperial College London, Exhibition Road, London SW7 2AZ, UK

3 Department of Imaging Sciences, Imperial College London, Exhibition Road, London SW7 2AZ, UK

4 Department of Surgery \& Cancer, Imperial College London, Exhibition Road, London SW7 2AZ, UK

\section{Corresponding author:}

Dr Meng-Xing Tang

Tel: +44 (0)20 75943664

Email: mengxing.tang@imperial.ac.uk

Postal address: Department of Bioengineering, Imperial College London, South Kensington Campus, London SW7 2AZ 
Abstract:

Tissue elasticity is closely related to the velocity of shear waves within biological tissue. Shear waves can be generated by an acoustic radiation force and tracked by, e.g., ultrasound or MRI measurements. This has been shown to be able to non-invasively map tissue elasticity in depth and has great potential in a wide range of clinical applications including cancer and cardiovascular diseases. In this study a highly sensitive optical measurement technique is proposed as an alternative way to track shear waves generated by the acoustic radiation force. A CCD camera was used to capture diffuse photons from tissue mimicking phantoms illuminated by a laser source at $532 \mathrm{~nm}$. CCD images were recorded at different delays after the transmission of an ultrasound burst, and were processed to obtain the time of flight for the shear wave. A differential measurement scheme involving generation of shear waves at two different positions was used to improve the accuracy and spatial resolution of the system. The results from measurements on both homogeneous and heterogeneous phantoms were compared to measurements from other instruments, and demonstrate the feasibility and accuracy of the technique for imaging and quantifying elasticity. The relative error in estimation of shear wave velocity can be as low as $5.5 \%$ with a spatial resolution of $2 \mathrm{~mm}$. The system is shown to be highly sensitive and is able to track shear waves propagating over several centimetres given the ultrasound excitation amplitude and the phantom material used in this study. It was also found that the reflection of shear waves from boundaries between regions with different elastic properties can cause significant bias in the estimation of elasticity, which also applies to other shear wave tracking techniques. This bias can be reduced at the expense of reduced spatial resolution.

Key words:

Ultrasound imaging, Elastography, Shear wave, Acoustic radiation force, Optical imaging, Ultrasound modulated optical tomography 


\section{Introduction}

Changes in the mechanical properties of soft tissue, such as shear elasticity (stiffness), are usually correlated with pathological changes. In vitro and in vivo studies show that normal breast tissue is several times softer than fibroadenoma tissue and carcinomas (Ophir et al. 1996). Manual palpation fails when lesions are small or deep in the body. Some lesions, such as breast tumours, are difficult to detect in standard ultrasound examinations due to the lack of echogenic properties (Garra et al. 1997).

In the past two decades a number of imaging techniques have been developed to noninvasively measure tissue elasticity in vivo (Greenleaf et al. 2003). The clinical potential of elasticity imaging has been demonstrated in imaging breast (Regner et al. 2006; Burnside et al. 2007), cardiovascular disease (Pislaru et al. 2004; Trahey et al. 2004), and fibrosis (Sandrin et al. 2003), amongst others.

In these elasticity imaging techniques tissues are deformed externally either by a low frequency vibrator (Parker et al. 1990) or static compression (Ophir et al. 1991), or internally by acoustic radiation force (ARF) (Sugimoto et al. 1990; Nightingale et al. 2002). Then tissue displacement within the tissue can be tracked by imaging techniques such as ultrasound (Lerner et al. 1990) or magnetic resonance imaging (Van Houten et al. 2003). However, these techniques typically only give relative measurements for tissue stiffness, partly due to the difficulty in obtaining accurate boundary conditions.

The tracking of shear waves generated by a transient ARF is an attractive method to quantify tissue stiffness (Sarvazyan et al. 1998). The acoustic radiation force (ARF) is a force resulting from the momentum transfer between the propagating ultrasound wave and the tissue. The $\mathrm{ARF}$ has the same direction as the ultrasound wave propagation, and its magnitude can be approximated by:

$$
E=\frac{2 a l}{\sigma},
$$


Where $F\left(\mathrm{~kg} /\left(\mathrm{s}^{2} \mathrm{~m}^{2}\right)\right)$, is the ARF, $a(\mathrm{~Np} / \mathrm{m})$ is the absorption coefficient of tissue, $I\left(\mathrm{~W} / \mathrm{m}^{2}\right)$ is the temporal average intensity and $c(\mathrm{~m} / \mathrm{s})$ is sound speed in the tissue. The force is capable of deforming soft tissues by micrometres, as described thoroughly by a recent review paper (Sarvazyan et al. 2010). When the ARF is applied to the tissue, shear waves are generated and propagate away from the excitation region. The velocity of shear waves can be directly related to the mechanical properties of tissue by:

$$
S=\sqrt{\frac{\mu}{\rho} ; \beta}=\frac{E}{2(1+\gamma)^{\prime}}
$$

Where $s$ is shear wave velocity, $\mu$ is shear modulus, $\rho$ is density, $E$ is Young's modulus and $\gamma$ is Poisson's ratio which is assumed to be 0.5 for incompressible tissues, providing the relationship $E=3 \mu$. Consequently, the Young's modulus can be directly related to shear wave velocity by:

$$
E=3 \rho s^{2},
$$

Young's modulus is an indicator of tissue stiffness, which can be obtained through this equation once shear wave velocity is known. Typically, there are two main ways to measure the shear wave speed: direct inversion of the Helmholtz equation (Oliphant et al. 2001; Nightingale et al. 2003; Bercoff et al. 2004) and time-of-flight measurement (Mclaughlin and Renzi 2006; Palmeri et al. 2008). However, these methods mainly use ultrasound to detect tissue displacement due to shear waves and the detection sensitivity is typically limited to tenths of a micron and above (Walker and Trahey, 1995).

Optical detection of diffused temporally coherent light from within tissue is known to be highly sensitive to tissue displacement. Tissue vibration as a result of diagnostic ultrasound excitation is typically at the level of hundreds of nanometres or below, and such vibration can be readily detected optically. This has been utilised in ultrasound modulated optical tomography (also called acousto-optic imaging) to image local tissue optical properties at millimetre to centimetre depth. Recent reviews of this topic can be found in (Wang 2004; 
Tang et al. 2010; Daniel S. Elson et al. 2011). Simulation results have shown that by using temporally coherent light and detecting the phase change of diffuse photons from tissue, tissue displacement at the level of nanometres can be detected ( $\mathrm{Li}$ et al. 2009). In this study, we propose the use of ARF based shear wave elasticity imaging using a modified ultrasoundmodulated optical tomography system with coherent light and a CCD camera (Li et al. 2010; Li et al. 2011).

\section{Methods}

\section{Experiment set up}

A broadband focused ultrasound transducer (Panametric NDT Videoscan 307, $5 \mathrm{~cm}$ focal length) was excited using a $1 \mathrm{~ms} 5 \mathrm{MHz}$ ultrasound burst with $6 \mathrm{MPa}$ peak negative pressure to generate shear waves in tissue mimicking phantoms. The attenuation coefficient of phantom is $0.16 \mathrm{~dB} \mathrm{~cm}^{-1} \mathrm{MHz}^{-1}$. A continuous $532 \mathrm{~nm} \mathrm{Nd:YAG} \mathrm{laser} \mathrm{beam} \mathrm{(Spectra-physics)}$ and a CCD camera (QImaging RetigaTM EXi fast 1394) was positioned perpendicular to the ultrasound axis (Figure 1). Whilst in our previous UOT system (Li et al. 2011) the optical axis was aligned perpendicular to the ultrasound axis and in line with the ultrasound focus, in this study the ultrasound axis was deliberately displaced from the optical axis (the distance between optical and acoustic axis is signified as DAO in Figure 1) to facilitate the tracking of shear wave velocity. The photons detected by the CCD passed through the optical detection volume defined by the laser scattering, and the resulting speckle pattern was processed to obtain the speckle contrast $C$ by dividing the standard deviation $\sigma$ of the $\mathrm{CCD}$ image intensity by its mean value $\{I\rangle(C=\sigma / G I))$. The value of $C$ is dependent on the motion within the optical region during the $\mathrm{CCD}$ exposure time (1.5 $\mathrm{ms}$ in this study). $\mathrm{C}$ equals one if there is no motion, and decreases due to tissue motion. Tissue motion causes a modification of the phase of the diffuse photons and subsequent blurring of the speckle image. The contrast difference $\Delta C$ was obtained by subtracting the image contrast measured before the ultrasound exposure to that measured afterwards. $\Delta C$ increases with the amplitude of tissue motion (Li et al. 2002). 
Optical measurements were repeated at various time points after the ultrasound exposure in order to track the propagation of the shear wave as a function of time, as seen in Figure $2 \mathrm{~b}$.

Time of flight-based calculation of shear wave velocity

Figure 2 illustrates the method for estimating the shear wave velocity. The acoustic axis (position A in Figure 2a) is separated from the optical axis (position O) by a certain distance (DAO). Shear waves are generated by transient ARF at the position A, and propagate toward the optical axis. Image contrast difference $\Delta C$ increases as the shear wave approaches the optical axis and will have a peak when the shear wave arrives at the optical axis (position O). Figure $2 \mathrm{~b}$ shows the expected change of $\Delta C$ with time, which gradually increases until the peak and then decreases as the shear wave propagates away from the optical region. The time to peak (TTP1 in Figure 2b) is related to the distance between acoustic position A and the optical detection axis $\mathrm{O}$ (DAO1 in Figure 2a) and the shear wave velocity.

Although shear wave velocity can be obtained by dividing DAO1 by TTP1 in Figure 2, in this study a differential technique is proposed involving ARF excitation at two positions (A and B in Figure 2a) in order to achieve improved spatial resolution and accuracy. If the position of the acoustic axis (and hence the shear wave origin) moves by $\Delta \mathrm{S}$ from position A to position B while the optical axis remains unchanged, the peak in $\Delta C$ will shift in time by $\Delta T$ (Figure 2b). Through knowledge of the time shift of the peak and the spatial distance between position $\mathrm{A}$ and $\mathrm{B}$, the local shear wave velocity between these two positions can be obtained by dividing $\Delta S$ by $\Delta T$.

The time to peak was estimated after fitting each time of flight curve shown in Figure $2 \mathrm{~b}$ with a cubic smoothing spline. Note that the decrease in the peak value of $\Delta C$ is due to the attenuation of shear wave. 


\section{Phantoms}

Homogeneous phantoms $(93.5 \mathrm{~mm} \times 42 \mathrm{~mm} \times 20 \mathrm{~mm})$ were created with three different agar concentrations $(0.8 \%, 1.0 \%$, and $1.2 \%$ agar) and $0.4 \%$ intralipid to simulate the scattering properties of biological tissue (Daoudi 2009). A heterogeneous phantom consisting of two different stiffnesses was made. The phantom $(93.5 \mathrm{~mm} \times 42 \mathrm{~mm} \times 20 \mathrm{~mm})$ was created by two conjoined slabs of the same dimensions (diagram in Figure 3), one of which contained $0.8 \%$ agar and another one contained $1.2 \%$ agar. The intralipid content was the same for both slabs, which was $0.4 \%$. Finally a phantom with a cylindrical mechanical inclusion was made. The background was made by $0.8 \%$ agar and inclusion was made by $1.2 \%$ agar. For both background and inclusion $0.4 \%$ intralipid was added, giving a reduced scattering coefficient of $5 \mathrm{~cm}^{-1}$ which is within the range of soft tissue. The radius of the inclusion was $3.5 \mathrm{~mm}$.

\section{Initial measurements and validation}

Initial measurements were made on the homogeneous phantoms to optimise the measurement parameters of the system. The optical axis (shown in Figure 1) was fixed and the ultrasound transducer was translated relative to it from $3 \mathrm{~mm}$ to $25 \mathrm{~mm}$ in steps of $1 \mathrm{~mm}$ (DAO in Figure 2a varied from $3 \mathrm{~mm}$ to $25 \mathrm{~mm}$ with $1 \mathrm{~mm}$ step size). By quantifying the time shift of the peak in the speckle contrast for different DAOs, the shear wave velocity was obtained. This experiment allowed us to evaluate the influence of, and determine the optimal values for, the following two measurement parameters: a) the distance between the acoustic and optical axes (DAO); and b) the distance between the two ultrasound positions.

To independently validate the Young's modulus obtained from this optical shear wave tracking method, a mechanical testing machine (Tinius Olsen H5KS) was used to compress the phantoms and the obtained stress-to-strain curves were used to calculate the corresponding Young's modulus using

$$
E=\frac{i}{A} \cdot \frac{g_{i}}{g_{i}}
$$


where $f$ is the compression stress measured with a force sensor, $\varepsilon$ is the phantom strain along the direction of compression, and $l$ and $A$ are the phantom height and the contact area of phantom surface and compression applicator respectively. The contact area between the holder and the phantom is much smaller than the phantom surface to avoid any change in the contact area during experiment. Preload was to keep the phantom from moving but was kept as small as $0.3 \mathrm{~N}$. For details on calculating Young's modulus using this method please see (Wells and Liang 2011).

\section{Measurement of the heterogeneous phantom}

$1 D$ scan

During the experiments on the heterogeneous phantoms (which consisted of a stiffer and softer section, as described above), only two DAOs (15 $\mathrm{mm}$ and $17 \mathrm{~mm}$ ) were used based on the initial study on homogeneous phantoms (results shown in the results section). Figure 3 illustrates the scanning scheme for heterogeneous phantoms. During the first scan, a $15 \mathrm{~mm}$ DAO was used and the phantom was translated along the direction indicated in the figure with $1 \mathrm{~mm}$ step size. For the second scan, the same phantom translation was repeated but the DAO was increased to $17 \mathrm{~mm}$. The scanning started with the ultrasound axis fully in the softer section of the phantom and was stepped towards the stiffer part. The measurement was repeated three times to evaluate the repeatability and noise.

$2 D$ scan

The phantom with a mechanical inclusion was scanned in $2 \mathrm{D}$ at a spatial step of $1 \mathrm{~mm}$ in both directions. A spatial map of the Young's modulus was built based on a single scan with DAOs of 15 and $17 \mathrm{~mm}$. A spatial smoothing Gaussian kernel (size: 5mm, sigma: $2 \mathrm{~mm}$ ) was used to post-process the image.

Finally in order to test the sensitivity of optical detection for weak shear waves, the homogeneous phantoms was measured with a fixed optical axis while the acoustic axis was moved in $1 \mathrm{~cm}$ steps up to $7 \mathrm{~cm}$ away from the optical axis. 


\section{Results}

\section{Initial measurements on homogenous phantoms and validation}

Experimental results are shown in Figure 4, where the $\mathrm{Y}$ axis presents different DAOs, the $\mathrm{X}$ axis is the time and the colour is the speckle contrast difference $(\Delta C)$. The maximum value of $\Delta C$ decreases as the DAO rises due to the attenuation of the shear wave. Different phantoms have different $\Delta C$ values due to their different stiffnesses. To further analyse the data and calculate the shear wave velocity, the TTP for each phantom at each DAO was found and plotted in Figure 5, where TTP is plotted against DAO. The relationship between different DAOs and TTPs was found to be linear when the acoustic axis was not close to optical axis, as expected. However, the slopes deviate from the linear trend for smaller DAOs for reasons described in the discussion section. The rate at which TTP changed for different transducer positions was evaluated using linear fitting for the Data from a $10 \mathrm{~mm}$ DAO onwards. The inverse of the slopes for these three fitted lines represent the shear wave speed of the respective material.

Table 1 compares the results when using the optical and mechanical methods. The Young's modulus was found to increase with the increase of agar concentration. The deviation between the optical and mechanical methods is small and is no more than $\pm 10 \%$. Note that the density was $1000 \mathrm{~kg} / \mathrm{m}^{3}$ for all phantoms.

To calculate the local shear wave velocity, two DAOs were required for our proposed differential method. The DAO determines the signal-to-noise ratio (SNR) for optical detection as the larger the DAO, the smaller the shear wave amplitude due to attenuation. On the other hand, the relationship between the DAO and TTP was nonlinear when DAO was small. Therefore $15 \mathrm{~mm}$ was chosen for one of the DAOs. For the other DAO, two conditions were considered: a) the difference of the two DAOs determines the spatial resolution of the system so the smaller the separation the better resolution; b) the accuracy of the shear wave velocity estimation improves with larger differences between the two DAOs. The results in Figure 6 
were calculated when the difference between the two DAOs was $1 \mathrm{~mm}, 2 \mathrm{~mm}$ and $3 \mathrm{~mm}$. It can be seen that the larger the separation, the smaller the standard deviations in the calculated shear wave velocity. The standard deviations for the hard phantom are calculated to be 0.47 , 0.20 , and 0.07 for the three differences in DAO respectively. Therefore, a balance must be made between estimation SNR and spatial resolution. In the subsequent study, the distance between the two ultrasound positions was chosen to be $2 \mathrm{~mm}$ and the two DAOs chosen for subsequent studies were $15 \mathrm{~mm}$ and $17 \mathrm{~mm}$.

\section{Results for the heterogeneous phantom}

Figure 7a shows the TTP while the phantom was scanned for the two chosen DAOs. For each curve in Figure 7a the TTP value varies depending on the elasticity properties between the acoustic and optical axis, while the difference between the two curves corresponds to the time for the shear wave to travel between the two DAOs. Based on this, the Young's modulus $(E)$ for the phantom was calculated and is shown in Figure $7 \mathrm{~b}$. It can be seen that the results are consistent with the expected values (dashed lines) when the ultrasound axis was away from the boundary between the soft and hard regions. The slight difference between the calculated Young's modulus and the expected value is likely due to variation in the fabrication of the phantom. When the ultrasound axis was close to the boundary between the soft and hard regions there is an unexpected maximum in both of the TTP curves. This causes first a trough and then a peak in the calculated Young's modulus. One possible reason for this is that shear wave reflection at the boundary constructively interferes with the original wave and causes a delay of the shear wave peak. It should be noted that the variations in each measurement in Figure 7 were less than $1 \%$ and hence the error bars were omitted.

\section{Investigation of shear wave reflection near the phantom soft-hard boundary}

In order to investigate whether the shear wave reflection was the cause for the maximum in Figure $7 \mathrm{a}$ and the estimation inaccuracy in elasticity in Figure $7 \mathrm{~b}$, the phantom was rotated 180 degrees horizontally so that the ultrasound axis began in the stiffer (1.2\% agar) part and 
the phantom was translated so that ultrasound axis moved into the softer $(0.8 \%$ agar $)$ part. In this case the shear wave reflection from the interface between the stiffer and softer parts should destructively interfere with the original shear wave thereby decreasing the TTP. These expected troughs were found experimentally as shown in Figure 8a. Calculation of Young's modulus again shows the estimation accuracy of our system (Figure 8b) except when the ultrasound axis was near to the phantom hard-soft boundary. In this case a peak was again found in the Young's modulus calculation but with no associated trough as in Figure 7b, which is also expected due to the shapes of the individual TTP curves in Figure 8a. The peak values of Young's modulus in the two experiments differ slightly (around $85 \mathrm{kPa}$ and $70 \mathrm{kPa}$ respectively). This is likely because the distance between the acoustic axis and the soft-hard boundary was not the same for the two experiments, resulting in changes of the overlap between reflected and original wave.

One method to reduce the boundary effect is to increase the separation of the two transducer positions to reduce the interference between the original and reflected shear waves. Figure 9 plots the results for $2 \mathrm{~mm}$ (15 $\mathrm{mm}$ and $17 \mathrm{~mm}$ DAOs) and $4 \mathrm{~mm}$ (15 mm and $19 \mathrm{~mm}$ DAOs) differences in DAO. As shown, the accuracy of Young's modulus measured at the boundary is increased by increasing the separation between the two transducer positions, at the expense of spatial resolution of the system.

\section{Results for $2 D$ scan}

Figure 10 shows the result for 2D scanning of the phantom with an inclusion. DAOs of 15 and $17 \mathrm{~mm}$ were used to obtain these data. The position of the inclusion is indicated by the dashed circle with which the obtained 2D image agrees well. The averaged estimated Young's modulus of the inclusion is approximately $55 \mathrm{kPa}$ in the figure which is higher than the $38 \mathrm{kPa}$ resulted from homogenous phantom measurements. The overestimation of the Young's modulus is likely due to the wave reflection at the boundary as well as the variations during the fabrication of the phantoms. 


\section{Sensitivity study}

To study the sensitivity of our optical detection of the shear wave at large DAOs we investigated a range of DAOs from 4 to $7 \mathrm{~cm}$. Figure 11 shows that even for $7 \mathrm{~cm} \mathrm{DAO,} \mathrm{the}$ shear wave propagation can still be detected with a high SNR.

\section{Discussion and Conclusions}

An experimental setup has been proposed that noninvasively measures the local velocity of a shear wave generated by the transient acoustic radiation force. This potentially offers a useful tool for measuring tissue elasticity in vivo. By studying homogeneous and heterogeneous phantoms, it has been shown that the proposed technique is feasible for tracking the shear wave propagation in tissue mimicking phantoms and to quantify local tissue elasticity. It should be noted that the proposed technique is a differential technique that should depend only on the difference in measurements for two ultrasound positions, and any heterogeneity between acoustic and optical axis should not affect the results.

Compared with existing techniques that mainly use ultrasound tracking, the proposed setup with optical tracking is potentially much more sensitive as it can detect tissue displacement of nanometres(Li et al. 2009), which potentially allows for a much more reliable estimation of tissue stiffness. In this study the optical measurements of tissue motion are still well above the noise even when the shear wave has propagated and been attenuated through $7 \mathrm{~cm}$ of the phantom. However it should be noted that the propagation distances over which shear wave is still detectable are dependent upon the initial displacement of the tissue and the material properties. Therefore further measurements in biological tissue are required to confirm the working range of the DAO for the system.

Compared with ultrasound detection of shear waves, optical measurements are less sensitive to tissue displacement at a depth beyond a few centimetres because light is highly scattered in tissues. However, the high sensitivity of the optical measurements means weak shear waves 
may still be detected as they propagate to the surface, that is to say, surface or subsurface optical measurements may detect a shear wave generated deep within tissue and this requires further study.

The presence of elasticity boundaries and shear wave reflections has a significant impact on the elasticity estimation and introduces bias. We have shown that this effect can be reduced at the expense of spatial resolution. It should be noted that this reflection phenomenon is common to all techniques that are based on tracking shear wave propagation. A recent study (Deffieux et al. 2011) has proposed some filtering techniques to reduce this effect, which would be interesting to test with our system.

The velocity estimation is biased when the ultrasound focus is close to the centre of the laser beam which is due to the finite size of the volume that the laser illuminates. When the DAO is small the shear wave is generated within the laser illuminated volume. In this case the optical detection tracks the shear wave propagation in both directions, which biases the time to peak measurements. This bias could also result from shear wave near-field diffraction effects, which means the shear wave needs to be a few millimetres outside the excitation region to observe a linear propagation trend.

In this study the CCD exposure time used was $1.5 \mathrm{~ms}$. This could be reduced to improve the maximum velocity detectable so that the system could be used to quantify very stiff materials. However a reduction in exposure time will also affect the SNR. Given the very good SNR we currently have, we believe there are plenty of rooms to reduce CCD exposure time. The spatial extent of the optical imaging volume will also affect the accuracy of shear wave measurements because the measurement is a weighted spatial average within the volume. The spatial extent of the optical beam and the weighting depends on many factors such as the scattering coefficient of the tissue and depth. 
In this study static tissue mimicking phantoms are used that did not take into account in vivo factors such as blood flow and tissue movement, which are likely to introduce extra noise to the optical measurements. Further studies are needed to evaluate this effect in vivo.

In conclusion, a highly sensitive shear wave elasticity imaging technique based on optical measurements was proposed to quantify tissue mechanical properties. The feasibility and accuracy of this technique has been studied. The reflection of shear waves near elasticity boundaries and the bias caused to the estimation of elasticity were studied and by using larger distances between acoustic excitations, this effect was reduced at the expense of system resolution

\section{Acknowledgements}

The authors would like to thank the UK EPSRC (Grant number: EP/H02316X/1) and the Royal Society for their financial support. Yi Cheng would like to thank China Scholarship Council for the financial support. 


\section{References}

Bercoff J, Tanter M, Fink M. Supersonic shear imaging, A new technique for soft tissue elasticity mapping. Ultrasonics, Ferroelectrics and Frequency Control, IEEE Transactions on 2004;51:396-409.

Burnside ES, Hall TJ, Sommer AM, Hesley GK, Sisney GA, Svensson WE, Fine JP, Jiang J, Hangiandreou NJ. Differentiating benign from malignant solid breast masses with US strain Imaging1. Radiology 2007;245:401-10.

Daniel S. Elson, Rui Li, Christopher Dunsby, Eckersley R, Tang M-X. Ultrasound-mediated optical tomography: A review of current methods. Interface Focus 2011;1:632-48.

Daoudi K. Detection and discrimination of optical absorption and shear stiffness at depth in tissue-mimicking phantoms by transient optoelastography. Appl Phys Lett 2009;94:154103.

Deffieux T, Gennisson JL, Bercoff J, Tanter M. On the effects of reflected waves in transient shear wave elastography. Ultrasonics, Ferroelectrics and Frequency Control, IEEE Transactions on 2011;58:2032-5.

Garra BS, Cespedes EI, Ophir J, Spratt RS, Zuurbier RA, Magnant CM, Pennanen M, F. Elastography of breast tissue lesions: Initial clinical results. Ridology 1997;202:79-86.

Greenleaf JF, Fatemi M, Insana M. Selected methods for imaging elastic properties of biological tissues. Annual Review of Biomedical Engineering 2003;5:57-78.

Joyce M, Daniel R. Shear wave speed recovery in transient elastography and supersonic imaging using propagating fronts. Inverse Problems 2006;22:681.

Lerner RM, Huang SR, Parker KJ. "Sonoelasticity" images derived from ultrasound signals in mechanically vibrated tissues. Ultrasound in Medicine\&Biology 1990;16:231-9.

Li J, Ku G, Wang LV. Ultrasound-modulated optical tomography of biological tissue by use of contrast of laser speckles. Appl Opt 2002;41:6030-5.

Li R, Elson DS, Dunsby C, Eckersley R, Tang M-X. A study on optical modulation signal and tissue displacement in ultrasound modulated optical tomography. SPIE Proceeding 2009; 
Li R, Elson DS, Dunsby C, Eckersley R, Tang M-X. Effects of acoustic radiation force and shear waves for absorption and stiffness sensing in ultrasound modulated optical tomography. Opt Express 2011;19:7299-311.

Li R, Song L, Elson DS, Tang M-X. Parallel detection of amplitude-modulated, ultrasoundmodulated optical signals. Opt Lett 2010;35:2633-5.

Nightingale K, McAleavey S, Trahey G. Shear-wave generation using acoustic radiation force: in vivo and ex vivo results. Ultrasound in Medicine\&Biology 2003;29:1715-23.

Nightingale K, Soo MS, Nightingale R, Trahey G. Acoustic radiation force impulse imaging: in vivo demonstration of clinical feasibility. Ultrasound in Medicine\&Biology 2002;28:22735.

Oliphant TE, Manduca A, Ehman RL, Greenleaf JF. Complex-valued stiffness reconstruction for magnetic resonance elastography by algebraic inversion of the differential equation. Magnetic Resonance in Medicine 2001;45:299-310.

Ophir J, Cespedes I, Carra B, Ponnekanti H, Huang Y, Maklad N. Elastography: Imaging the elastic properties of soft tissues with ultrasound. EurJUltrasound 1996;3:47-70.

Ophir J, Céspedes I, Ponnekanti H, Yazdi Y, Li X. Elastography: A quantitative method for imaging the elasticity of biological tissues. Ultrasonic Imaging 1991;13:111-34.

Palmeri ML, Wang MH, Dahl JJ, Frinkley KD, Nightingale KR. Quantifying hepatic shear modulus in vivo using acoustic radiation force. Ultrasound in Medicine\&Biology 2008;34:546-58.

Parker KJ, Huang SR, Musulin RA, Lerner RM. Tissue response to mechanical vibrations for “sonoelasticity imaging”. Ultrasound in Medicine\&Biology 1990;16:241-6.

Pislaru C, Bruce CJ, Anagnostopoulos PC, Allen JL, Seward JB, Pellikka PA, Ritman EL, Greenleaf JF. Ultrasound strain imaging of altered myocardial stiffness. Circulation 2004;109:2905-10.

Regner DM, Hesley GK, Hangiandreou NJ, Morton MJ, Nordland MR, Meixner DD, Hall TJ, Farrell MA, Mandrekar JN, Harmsen WS, Charboneau JW. Breast lesions: Evaluation with us strain imaging-clinical experience of multiple observers1. Radiology 2006;238:425-37. 
Sandrin L, Fourquet B, Hasquenoph J-M, Yon S, Fournier C, Mal F, Christidis C, Ziol M, Poulet B, Kazemi F, Beaugrand M, Palau R. Transient elastography: A new noninvasive method for assessment of hepatic fibrosis. Ultrasound in Medicine\&Biology 2003;29:1705-13. Sarvazyan AP, Rudenko OV, Nyborg WL. Biomedical applications of radiation force of ultrasound: historical roots and physical basis. Ultrasound Med Biol 2010;36:1379-94.

Sarvazyan AP, Rudenko OV, Swanson SD, Fowlkes JB, Emelianov SY. Shear wave elasticity imaging: A new ultrasonic technology of medical diagnostics. Ultrasound in Medicine\& Biology 1998;24:1419-35.

Sugimoto T, Ueha S, Itoh K. Tissue hardness measurement using radiation force of focused ultrasound. In Proc. IEEE Ultrason Symp 1990;NY: IEEE:1377-80.

Tang MX, Elson DS, Li R, Dunsby C, Eckersley RJ. Photoacoustics, thermoacoustics, and acousto-optics for biomedical imaging. Proceedings of the Institution of Mechanical Engineers, Part H: Journal of Engineering in Medicine 2010;224:291-306.

Trahey GE, Palmeri ML, Bentley RC, Nightingale KR. Acoustic radiation force impulse imaging of the mechanical properties of arteries: In vivo and ex vivo results. Ultrasound in Medicine\&Biology 2004;30:1163-71.

Van Houten EEW, Doyley MM, Kennedy FE, Weaver JB, Paulsen KD. Initial in vivo experience with steady-state subzone-based MR elastography of the human breast. Journal of Magnetic Resonance Imaging 2003;17:72-85.

Walker WF, Trahey GE. A fundamental limit on delay estimation using partially correlated speckle signals. Ultrasonics, Ferroelectrics and Frequency Control, IEEE Transactions on $1995 ; 42: 301-8$.

Wang LV. Ultrasound-mediated biophotonic imaging: A review of acousto-optical tomography and photo-acoustic tomography Disease Markers 2004;19:123-38.

Wells PNT, Liang H-D. Medical ultrasound: Imaging of soft tissue strain and elasticity. Journnal of the royal society 2011;8:1521-49. 
Figure 1 Experiment setup. DAO: the distance between optical and acoustic axis, which can be adjusted by using the translation stage (not shown in the Figure), AMP: power amplifier, WG: waveform generator and DG: delay generator. The ultrasound absorbing material minimises the ultrasound reflection. The size of the tank is $30 \mathrm{~cm} \times 20 \mathrm{~cm} \times 7.8 \mathrm{~cm}$. For more details for this set-up, please see (Li et al. 2010; Li et al. 2011).

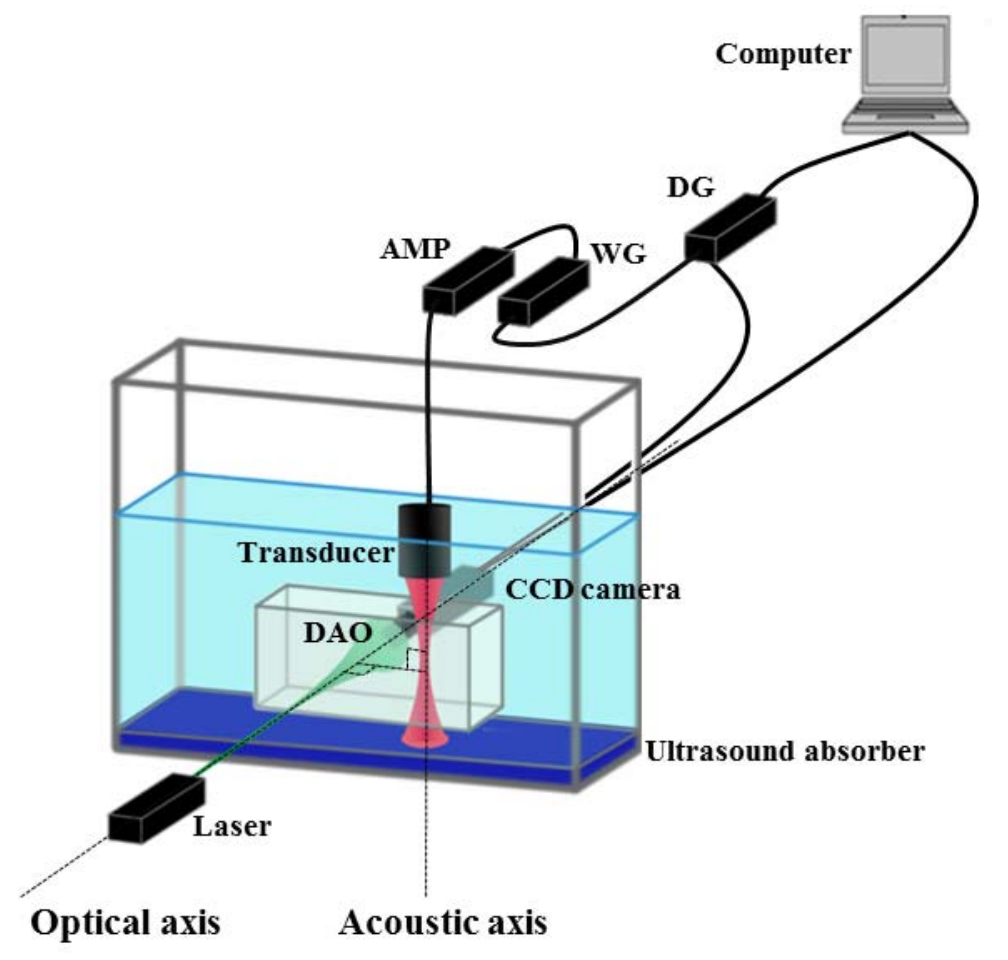


Figure 2 (a) Diagram of measurement principle. (b) Expected signals (optical contrast difference) as a function of time for ultrasound positions A and B. By finding the ratio of $\Delta \mathrm{S}$ and $\Delta \mathrm{T}$, the average velocity between $\mathrm{A}$ and $\mathrm{B}$ can be obtained. Position $\mathrm{O}$ indicates the optical axis position, A and B are two different acoustic axis positions.
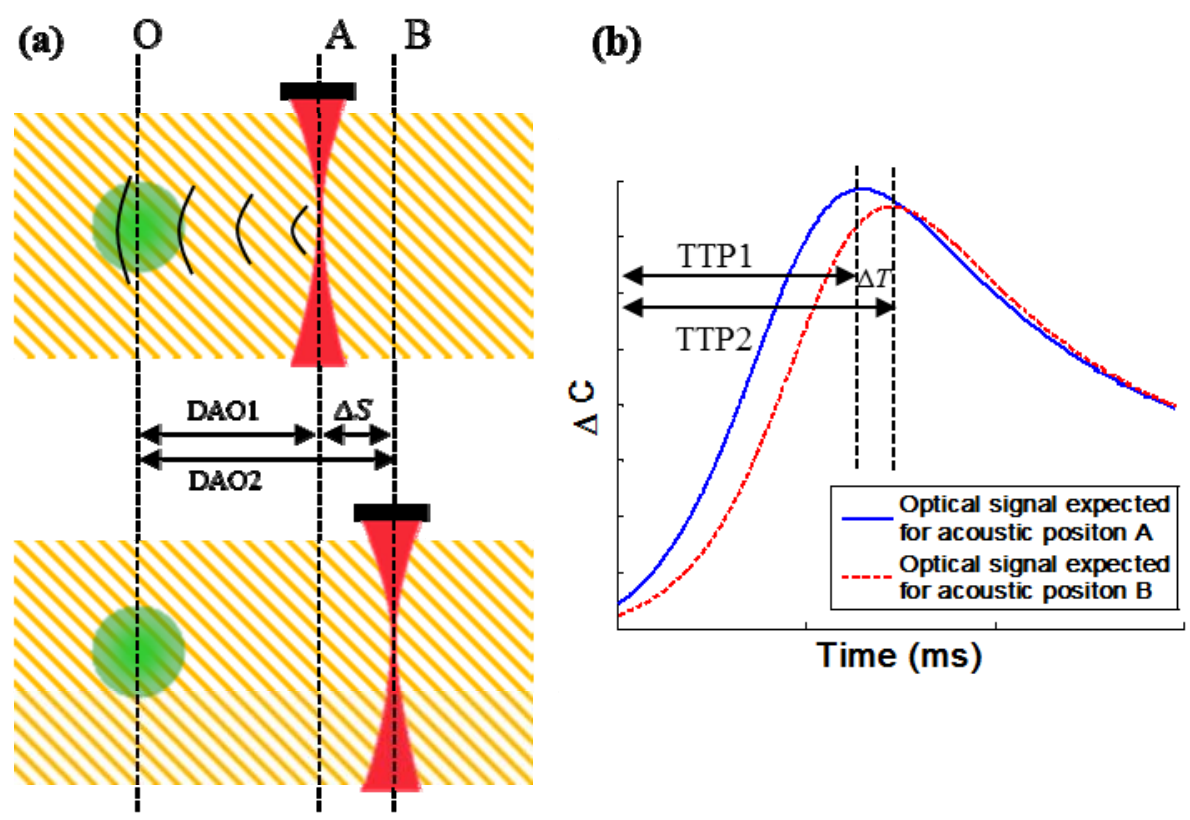

Time (ms) 
Figure 3 Scanning scheme for the heterogeneous phantom. Position $\mathrm{O}$ indicates the starting position for optical axis in two scanning runs. A and B are two different acoustic axis positions.

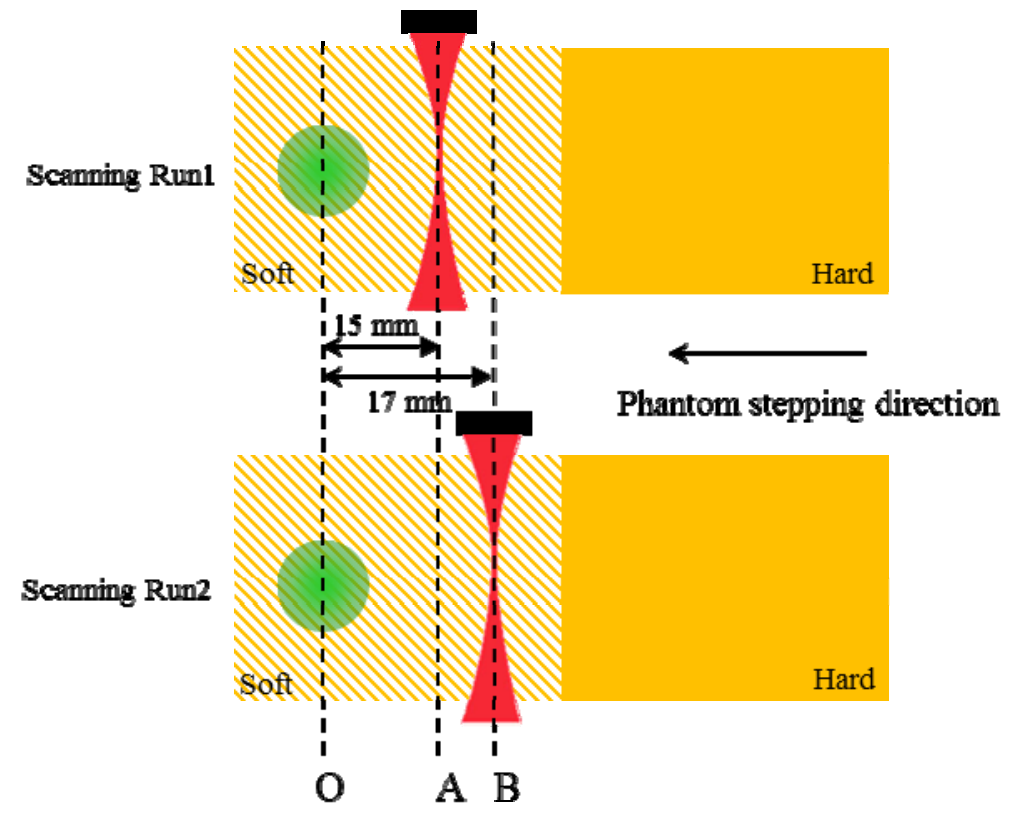

Figure 4 Optical contrast difference $(\Delta C)$ as a function of time and DAO in three homogenous phantoms of different stiffness.
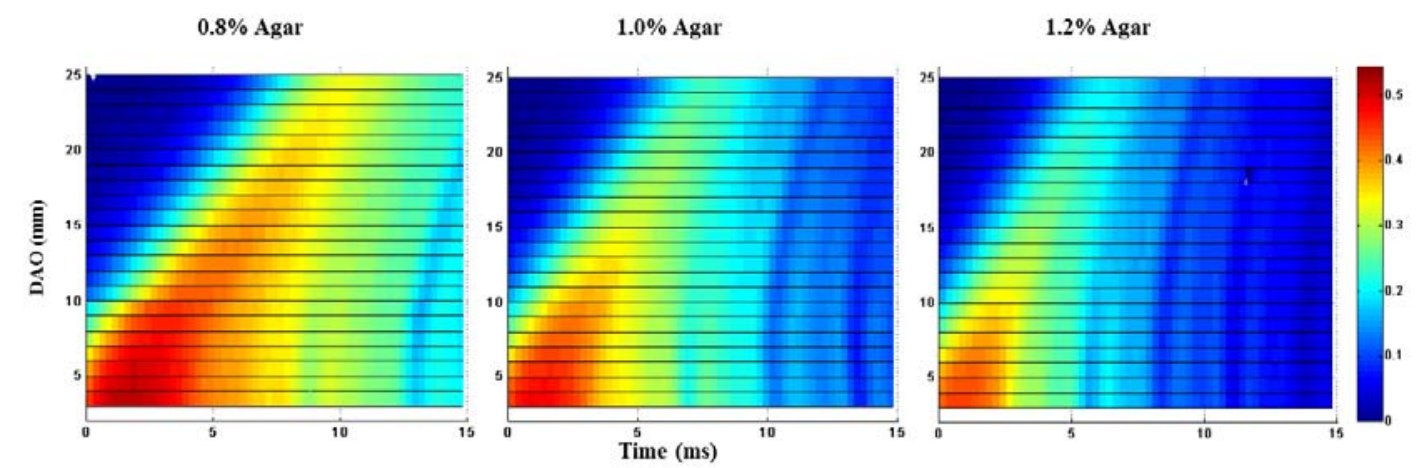
Figure 5 Time to peak (TTP) as a function of DAO in three homogenous phantoms of different stiffness. Young's Modulus can be calculated as the inverse of the slopes.

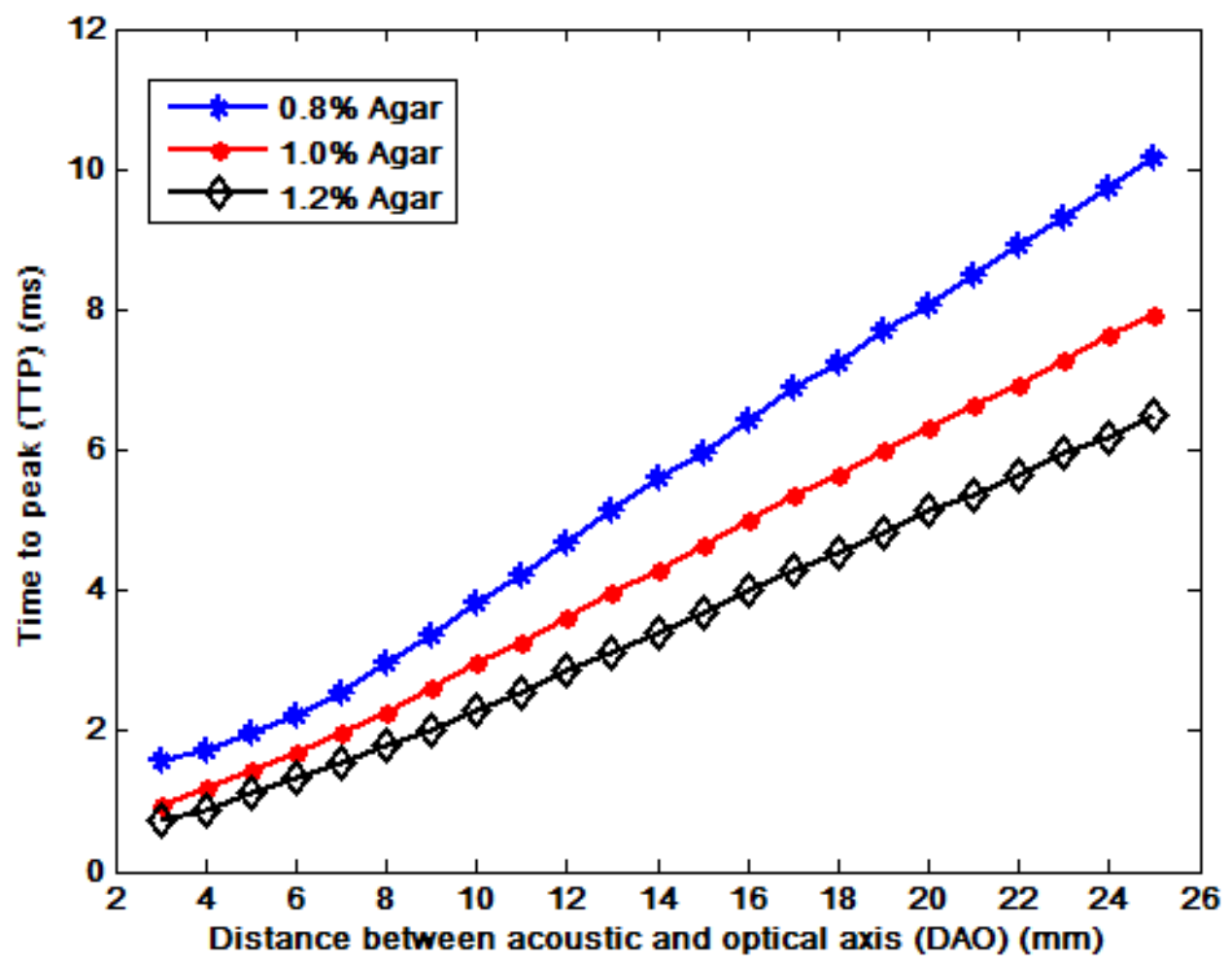


Figure 6 Estimated shear wave velocities in different phantoms with three separation distances between two DAOs. It can be seen that increasing the distance between the two DAOs reduces the variations of the measurements.

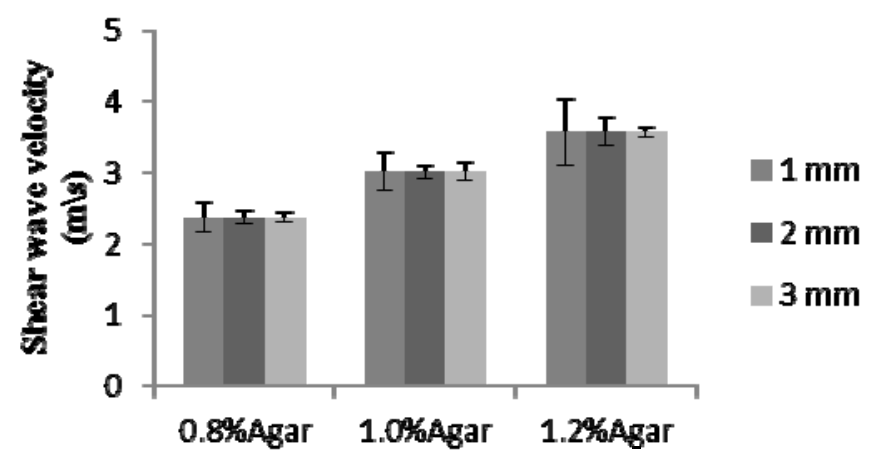

Figure 7 Scanning results of the heterogeneous phantom. (a) Time to peak (TTP) and (b) calculated Young's modulus across the phantom.

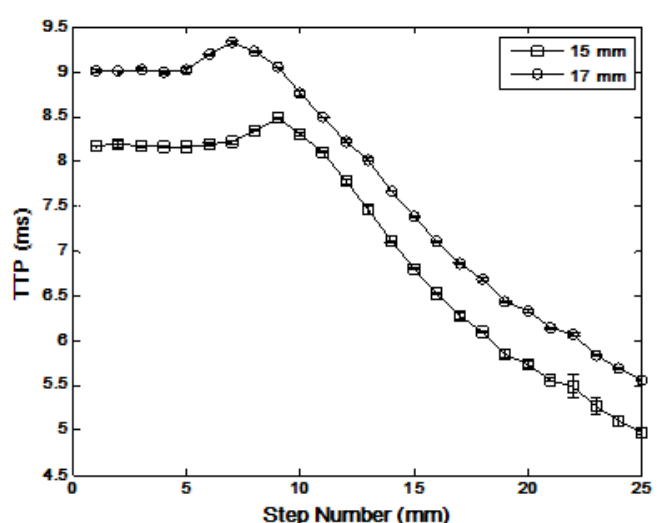

(a)

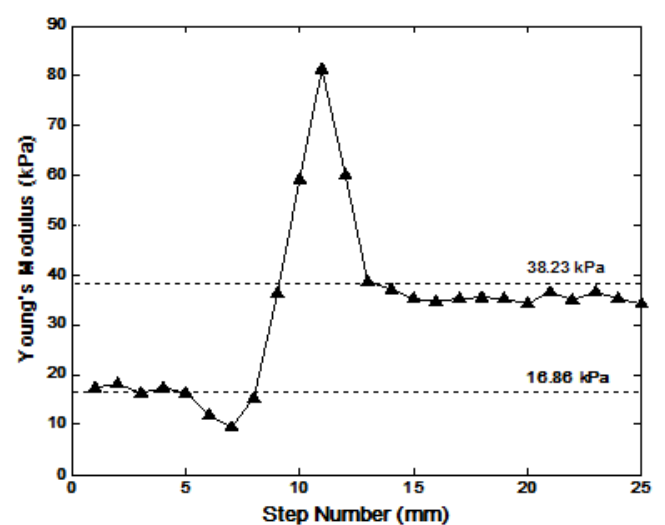

(b) 
Figure 8 Scanning results of the heterogeneous phantom that was turned 180 degrees horizontally. (a) Time to peak (TTP) and (b) calculated Young's modulus across the phantom.

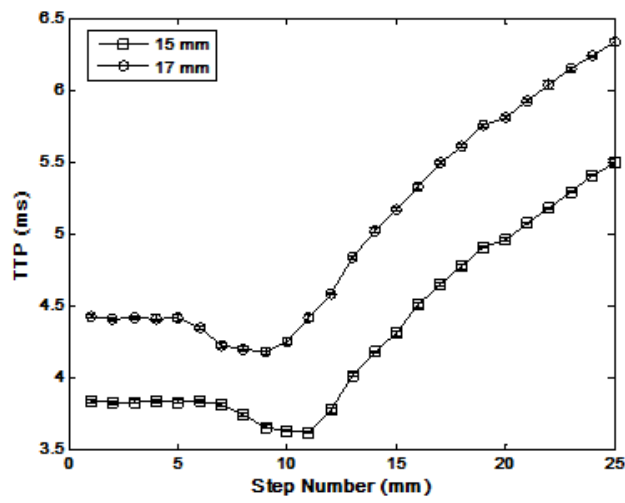

(a)

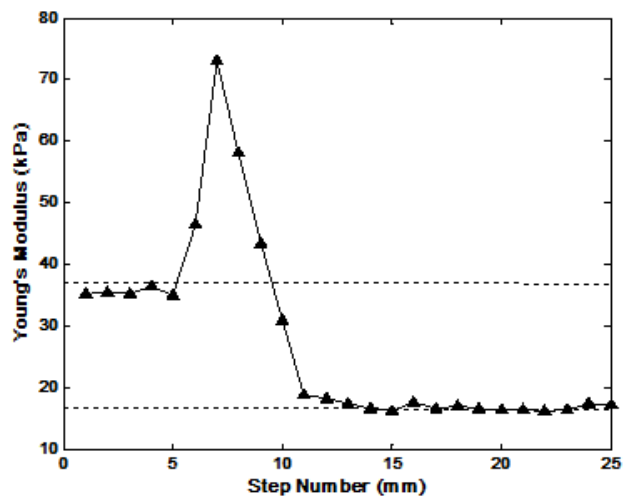

(b)

Figure 9 Scanning results of the heterogeneous phantom with $2 \mathrm{~mm}$ and $4 \mathrm{~mm}$ separation distance for the two DAOs. It can be seen that accuracy of estimation in Young's modulus is improved at the expense of image resolution when the separation of two DAOs increases.

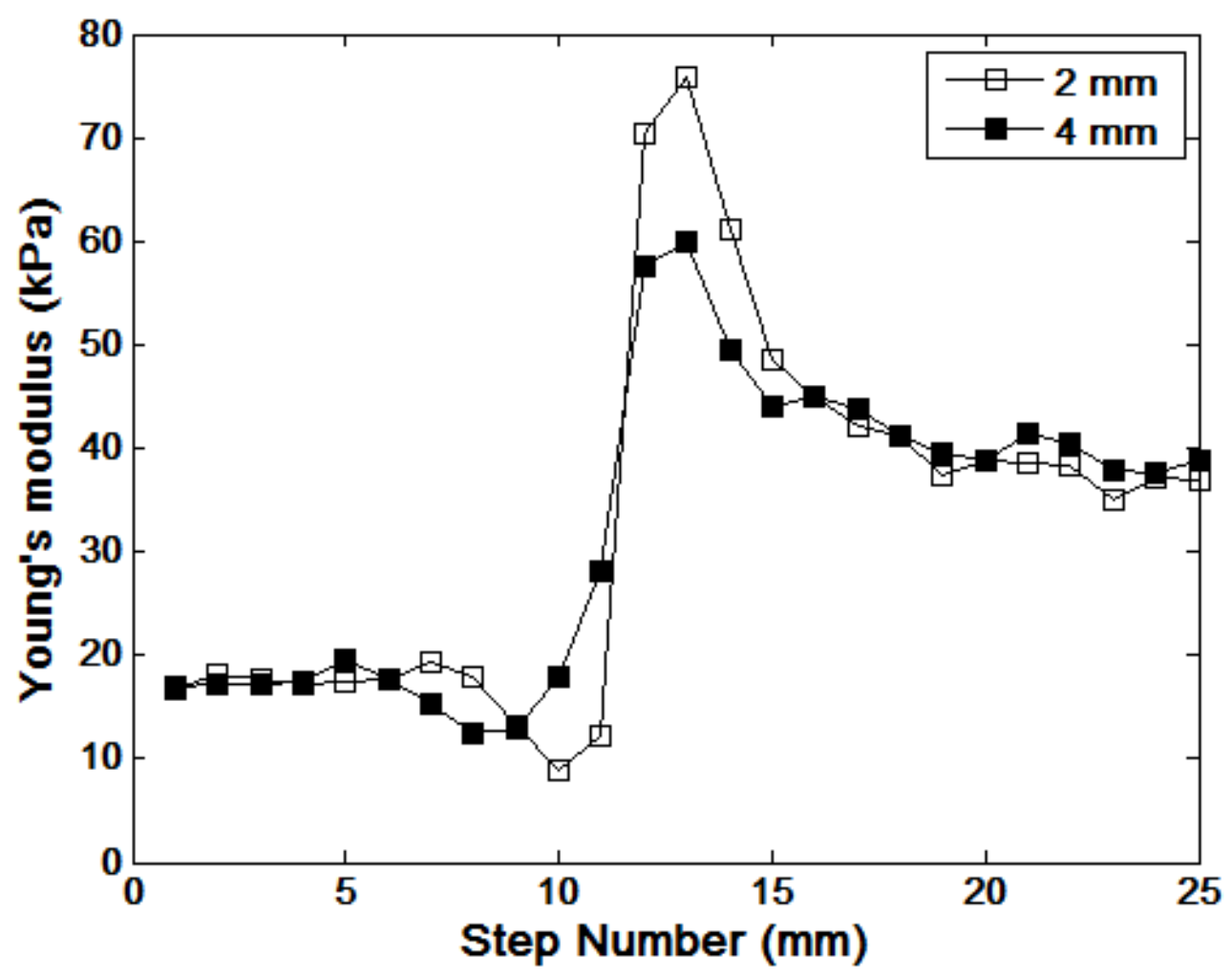


Figure 10 Two dimensional scan of a phantom with a cylindrical stiff inclusion $(3.5 \mathrm{~mm}$ in radius). The figure only shows part of the phantom with the inclusion.




Figure 11 Image contrast difference $(\Delta C)$ changes as the function of time with various DAOs ranging between $4 \mathrm{~cm}$ and $7 \mathrm{~cm}$.

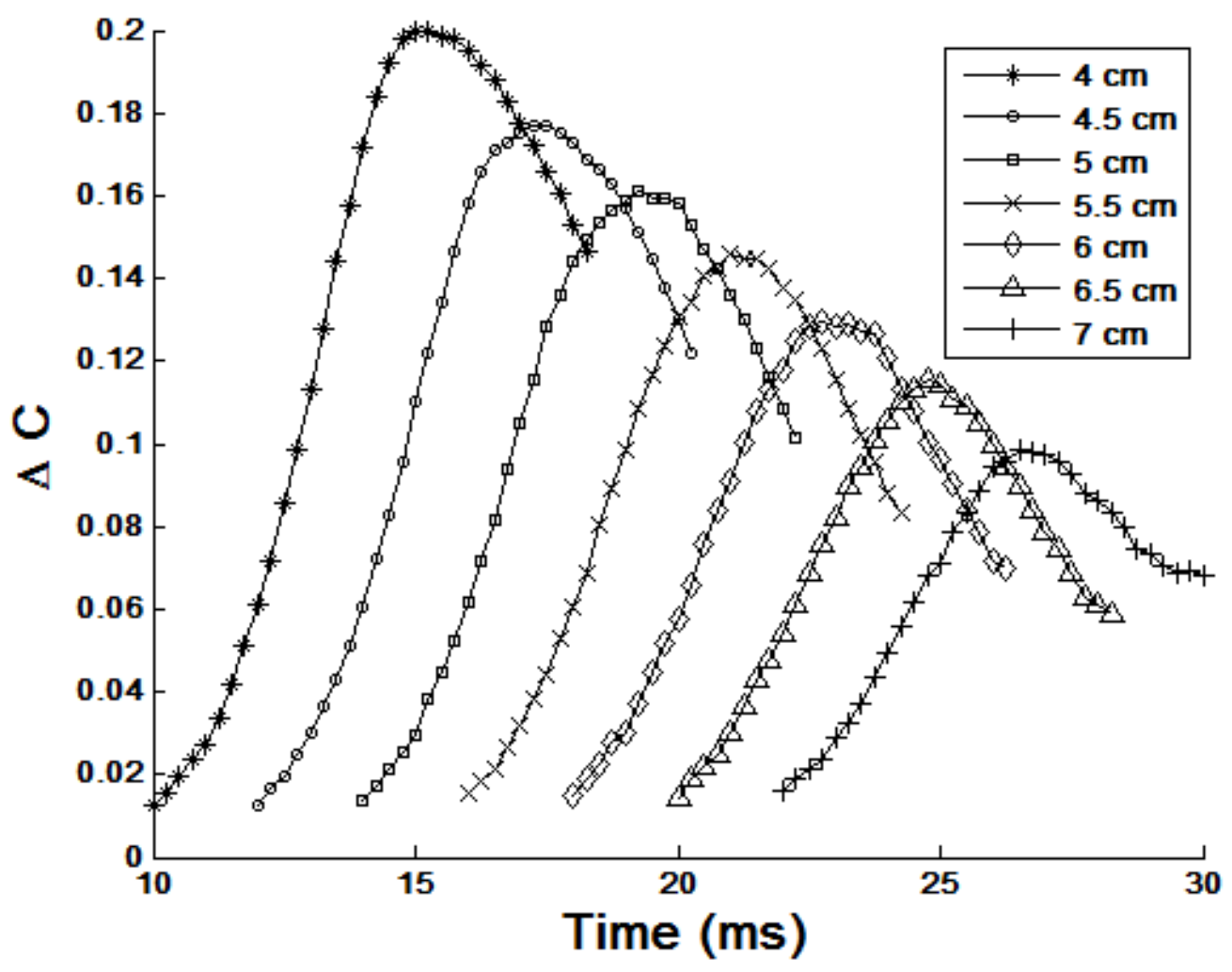


Table 1 Young's modulus of phantoms measured with a mechanical testing machine and optical detection

\begin{tabular}{ccccc}
\hline Phantoms & $\begin{array}{l}\text { Shear wave velocity } \\
\text { (Optical method) }\end{array}$ & $\begin{array}{l}\text { Young's modulus } \\
\text { (Optical method) }\end{array}$ & $\begin{array}{c}\text { Young's modulus } \\
\text { (Mechanical test) }\end{array}$ & Deviation \\
\hline $\mathbf{0 . 8 \%}$ agar & $2.37 \mathrm{~m} / \mathrm{s}$ & $16.86 \mathrm{kPa}$ & $17.96 \mathrm{kPa}$ & $6.52 \%$ \\
$\mathbf{1 . 0 \%}$ agar & $3.01 \mathrm{~m} / \mathrm{s}$ & $27.18 \mathrm{kPa}$ & $26.52 \mathrm{kPa}$ & $2.47 \%$ \\
$\mathbf{1 . 2 \%}$ agar & $3.57 \mathrm{~m} / \mathrm{s}$ & $38.23 \mathrm{kPa}$ & $35.47 \mathrm{kPa}$ & $9.78 \%$ \\
\hline
\end{tabular}

